\title{
Effect of Using Local Intrawound Vancomycin Powder in Addition to Intravenous Antibiotics in Posterior Lumbar Surgery: Midterm Result in a Single-Center Study
}

\author{
Gun-III Lee', Koang Hum Bak ${ }^{1}$, Hyoung-Joon Chun ${ }^{1}$, Kyu-Sun Choi ${ }^{2}$ \\ ${ }^{l}$ Department of Neurosurgery, Hanyang University Medical Center, Hanyang University College of Medicine, Seoul, \\ ${ }^{2}$ Department of Neurosurgery, Asan Medical Center, University of Ulsan College of Medicine, Seoul, Korea
}

\begin{abstract}
Objective: We conducted this study to report the efficacy of local application of vancomycin powder in the setting of surgical site infection (SSI) of posterior lumbar surgical procedures and to figure out risk factors of SSIs.

Methods: From February 2013 to December 2013, SSI rates following 275 posterior lumbar surgeries of which intrawound vancomycin powder was used in combination with intravenous antibiotics (Vanco group) were assessed. Compared with 296 posterior lumbar procedures with intravenous antibiotic only group from February 2012 to December 2012 (non-Vanco group), various infection rates were assessed. Univariate and multivariate analysis to figure out risk factors of infection among Vanco group were done. Results: Statistically significant reduction of SSI in Vanco group (5.5\%) from non-Vanco group (10.5\%) was confirmed $(p=0.028)$. Mean follow-up period was 8 months. Rate of acute staphylococcal SSIs reduced statistically significantly to $4 \%$ compared to $7.4 \%$ of non-Vanco group ( $\mathrm{p}=0.041)$. Deep staphylococcal infection decreased to 2 compared to 8 and deep methicillin-resistant Staphylococcus aureus infection also decreased to 1 compared to 5 in non-Vanco group. No systemic complication was observed. Statistically significant risk factors associated with SSI were diabetes mellitus, history of cardiovascular disease, length of hospital stay, number of instrumented level and history of previous surgery.

Conclusion: In this series of 571 patients, intrawound vancomycin powder usage resulted in significant decrease in SSI rates in our posterior lumbar surgical procedures. Patients at high risk of infection are highly recommended as a candidate for this technique.
\end{abstract}

Key Words: Surgical wound infection $\cdot$ Vancomycin $\cdot$ Topical administration $\cdot$ Staphylococcal infection

\section{INTRODUCTION}

As well known, surgical site infection (SSI) following spine surgery is devastating with significant increase in hospital stay, health care costs and morbidities ${ }^{3,9)}$. Though incidence is reported to be ranging from $1.9 \%-13 \%$, these days, SSIs are the most frequent hospital-acquired infection outweighing urinary catheter infections, ventilator acquired pneumonias, central line-related infections, and Clostridium difficile infections ${ }^{2,8,15,16,19,21)}$. Most of these SSIs require additional surgical treatments for exploration, sampling and debridement.

In spine surgery, operative wound infections are known to be caused by gram-positive organisms, primarily Staphylococcus

\footnotetext{
- Received: February 23, 2016 • Revised: May 4, 2016

- Accepted: June 2, 2016

Corresponding Author: Koang Hum Bak

Department of Neurosurgery, Hanyang University Medical Center,

Hanyang University College of Medicine, 222-1 Wangsimni-ro,

Seongdong-gu, Seoul 04763, Korea

Tel: +82-2-2290-8496, Fax: +82-2-2281-0954

E-mail: pkh2325@hanyang.ac.kr

$@$ This is an open access article distributed under the terms of the Creative Commons Attribution Non-Commercial License (http://creativecommons.org/licenses/by-nc/4.0/) which permits unrestricted non-commercial use, distribution, and reproduction in any medium, provided the original work is properly cited.
}

aureus and Staphylococcus epidermidis ${ }^{10,20)}$. Correspondingly, usage of broad spectrum antimicrobial drugs such as cefazolin has been a routine practice to prevent SSI for several decades ${ }^{13,27}$. Lamentably, the proportion of infections caused by methicillin-resistant staphylococci has been rising, thus decreasing the efficacy of cephalosporins for preventing $\mathrm{SSIs}^{26}$.

Both patient-related and operation-related risk factors contribute to the development of SSIs. Patient-related risk factors include obesity, diabetes and an immunocompromised state, while operation-related factors consist of multilevel surgery, use of instrumentation, revision surgery, and large intraoperative blood loss ${ }^{4,17,18)}$.

Recently, direct use of intrawound vancomycin powder has shown to decrease SSI rates ${ }^{14,16,25)}$. The aim of present study is to assess incidence, risk factors and outcome of SSIs between patients with or without local intrawound vancomycin powder at the surgical site, in addition to standard intravenous prophylaxis. And also, to evaluate risk factors for SSI in setting of local vancomycin instillation.

\section{MATERIALS AND METHODS}

\section{Patients and Study Design}

All operative procedures were performed by 2 senior sur- 
geons (KHB and HJC) in a single center. From February 2012 to December 2013, consecutive 571 patients undergoing posterior lumbar surgery at Hanyang University Medical Center were enrolled in the present study. Patients were selected according to following inclusion and exclusion criteria. We reviewed the medical charts and radiographic materials of the above patients, retrospectively.

Patients were divided into 2 groups according to the date of operation. Prior to February of 2013, all patients undergoing posterior lumbar surgery received standard prophylactic intravenous antibiotics only. Beginning in February of 2013, all patients undergoing posterior lumbar surgery received between $1 \mathrm{~g}$ of vancomycin powder applied directly to the wound just prior to closure, in addition to standard prophylactic intravenous antibiotics.

\section{Exclusion Criteria}

Following patients were excluded from this analysis: (1) previous history of infections at the surgical site; (2) biopsy procedure; (3) patients with a postoperative follow-up time of less than 12 weeks; (4) patients allergic to vancomycin; (5) patients undergoing minimal invasive spine surgery; (6) surgery due to trauma related lesions.

\section{Variables and Data Sources}

Following selection of patients, their data were collected including patients' status, operative characteristics and demographic factors.

Patients' status included mean American Society of Anesthesiologists (ASA) grade and hospitalized days until discharge or SSI development. Operative characteristics included presence of previous surgery, postoperative diagnosis, hemovac drain usage, operative time, number of levels instrumented, whether decompressed or not and estimated blood loss (mL). Demographic factors assessed patient's sex, age, adult body mass index (BMI) and medical comorbidities (hypertension, diabetes mellitus, history of respiratory and cardiovascular disease, and smoking habit).

Other measures including number of neurosurgical residents involved in the surgery and method of hair removal (whether clipper or razor usage) were indifferent between 2 groups. Massive surgical site irrigation with normal saline and double glove technique were routinely used in all operative procedures.

\section{Protocols}

Standard prophylactic intravenous antibiotics for spinal surgery included $2 \mathrm{~g}$ of cefotetan given intravenously within one hour prior to incision and with repeat dosing every 4 hours during surgery. Postoperatively, patients were given $2 \mathrm{~g}$ of cefotetan intravenously every 12 hours for 5 days.

We defined an SSI as an infection occurring within 12 weeks following the operation, requiring an additional operation (i.e., an irrigation and debridement) and having positive wound cultures. Infections were further subdivided into superficial (occurring above the lumbosacral fascia) or deep (beneath the lumbosacral fascia) based on the wound culture reports and operative reports.

\section{Statistical Analysis}

Variables suitable for nominal scale were converted into categorical variables by dichotomization. The chi-square, Fisher exact test were used when appropriate for group comparisons. Categorized data (gender, age, obesity [adult BMI above 25 $\mathrm{kg} / \mathrm{m}^{2}$ ], hypertension, diabetes mellitus, smoking, history of respiratory diseases and cardiovascular diseases, estimated blood loss over $1,000 \mathrm{~mL}$, whether decompression was done or not, history of previous surgery, usage of hemovac) were analyzed using Fisher exact and/or chi-square tests. ASA score, length of hospital stay, operative time and number of level instrumented were analyzed using independent t-test. Univariate analysis was performed to determine associations between the SSI and potential covariates. Covariates with a p-value of $<0.05$ were entered into a binary logistic regression. We considered a p-value of $<0.05$ to be significant for all analyses.

\section{RESULTS}

\section{Patient Characteristics}

Since February 2012 to December 2013, total 693 consecutive posterior lumbar surgeries have been performed. Among them, 122 patients were excluded due to trauma-related lesions $(n=96)$, loss of follow-up within 12 weeks $(n=17)$, biopsy procedure $(n=3)$, allergic to vancomycin $(n=2)$, and history of infection $(n=4)$.

The patient group who underwent surgery from February 2013 to December 2013 and received intrawound vancomycin powder in addition to the standard antimicrobial prophylaxis was defined as Vanco group $(n=275)$. The other group who had surgery from February 2012 to December 2012 and did not receive intrawound vancomycin powder was named as non-Vanco group $(n=296)$. Demographic factors of patients did not show significant difference between 2 groups except mean ASA grade $(p<0.05)$. Operative data also were unable to show statistical significant difference between the 2 groups. The summaries of demographic and operative data are presented in Tables 1 and 2, respectively.

\section{Incidence of SSIs}

Among 571 patients, the overall incidence of SSIs was 8.1\% (46 of 571). Total staphylococcal infection was present in 33 patients (5.8\%) with deep methicillin-resistant Staphylococcus 
Local Intrawound Vancomycin in Posterior Lumbar Surgery

Table 1. Demographic characteristics of patients with or without intrawound vancomycin (Vanco) powder

\begin{tabular}{llll}
\hline \hline Characteristic & $\begin{array}{c}\text { Vanco } \\
(\mathrm{n}=275)\end{array}$ & $\begin{array}{c}\text { Non-Vanco } \\
(\mathrm{n}=296)\end{array}$ & p-value \\
\hline Age (yr) & $50.2 \pm 18.3$ & $52.1 \pm 19.8$ & \\
Age $>60$ yr (\%) & 40.7 & 42.9 & 0.599 \\
Female sex (\%) & 53.5 & 51.4 & 0.287 \\
Adult BMI (kg/m $\left.{ }^{2}\right)$ & $29.7 \pm 7.0$ & $31.2 \pm 7.5$ & \\
Obesity (\%) & & & \\
$\quad$ BMI >25 kg/m & 36.7 & 64.9 & 0.602 \\
Comorbidities (\%) & & & \\
$\quad$ Diabetes mellitus & 16.7 & 15.9 & 0.402 \\
$\quad$ Cardiovascular disease & 26.5 & 28.7 & 0.625 \\
$\quad$ Respiratory disease & 15.6 & 12.8 & 0.470 \\
$\quad$ Hypertension & 42.5 & 44.6 & 0.513 \\
$\quad$ Smoking & 19.6 & 22.0 & 0.496 \\
ASA grade & $2.3 \pm 0.5$ & $2.5 \pm 0.6$ & 0.040 \\
No. of days hospitalized & $10.3 \pm 5.3$ & $11.2 \pm 4.9$ & 0.106 \\
Follow-up (mo) & 8 & 11 & \\
\hline
\end{tabular}

Values are presented as mean \pm standard deviation unless otherwise indicated.

BMI, body mass index; ASA, American Society of Anesthesiologists.

t-test.

Table 2. Operative characteristics of patients with or without intrawound vancomycin (Vanco) powder

\begin{tabular}{lrrr}
\hline \hline Characteristic & $\begin{array}{c}\text { Vanco } \\
(\mathrm{n}=275)\end{array}$ & $\begin{array}{c}\text { Non-Vanco } \\
(\mathrm{n}=296)\end{array}$ & p-value \\
\hline $\begin{array}{l}\text { Estimated blood loss (mL), } \\
\text { mean } \pm \text { SD }\end{array}$ & $725 \pm 810$ & $680 \pm 800$ & \\
$\quad$ Blood loss >1,000 mL (\%) & 32.7 & 31.4 & 0.391 \\
Operative time (min) & 192.7 & 178.4 & 0.298 \\
No. of levels instrumented & 0.7 & 0.8 & 0.17 \\
Decompression (\%) & 87.3 & 93.2 & 0.478 \\
Previous surgery (\%) & 23.6 & 25.3 & 0.384 \\
Hemovac drain usage (\%) & 86.2 & 86.1 & 0.557 \\
\hline
\end{tabular}

SD, standard deviation.

t-test.

aureus (MRSA) infection in 6 patients and deep staphylococcal infection in 10 patients. Nonstaphylococcal infection was found in 11 patients (1.9\%). In Vanco group, staphylococcal infection was present in 11 patients with deep MRSA infection in 1 case. Nonstaphylococcal infection was observed in 4 patients. In total, 15 patients presented with SSIs in Vanco group. On the other hand, in non-Vanco group, acute SSIs developed in total of 29 patients, 8 of which were staphylococcal infection. Deep MRSA infection was confirmed in 5 patients and nonsta-
Table 3. Rates of infections

\begin{tabular}{lccc}
\hline \hline Characteristic & $\begin{array}{c}\text { Vanco } \\
(\mathrm{n}=275)\end{array}$ & $\begin{array}{c}\text { Non-Vanco } \\
(\mathrm{n}=296)\end{array}$ & p-value" \\
\hline Total infection & 15 & 31 & 0.028 \\
Total staphylococcal & 11 & 24 & 0.041 \\
Deep staphylococcal & 2 & 8 & 0.109 \\
Deep MRSA & 1 & 5 & 0.218 \\
Non-staphylococcal & 4 & 7 & 0.548 \\
\hline Vanco, vancomycin; MRSA, Methicillin-resistant Staphyloco- \\
ccus aureus. \\
"Chi-square/Fisher exact text.
\end{tabular}

Table 4. Univariate statistical analysis of potential risk factors

\begin{tabular}{lcr}
\hline \hline Variable & OR (95\% CI) & p-value \\
\hline Age & $1.714(0.603-4.871)$ & 0.312 \\
Sex & $1.777(0.615-5.137)$ & 0.288 \\
Obesity & $3.714(1.232-11.196)$ & 0.020 \\
Diabetes mellitus & $4.958(1.701-14.455)$ & 0.003 \\
History of cardiovascular disease & $3.429(1.197-9.822)$ & 0.022 \\
History of respiratory disease & $1.375(0.371-5.092)$ & 0.634 \\
Hypertension & $0.895(0.309-2.588)$ & 0.838 \\
Smoking & $2.153(0.704-6.583)$ & 0.179 \\
ASA & $1.433(0.618-3.325)$ & 0.402 \\
Hospital stay & $1.296(1.176-1.429)$ & $<0.001$ \\
Blood loss & $4.500(1.490-13.591)$ & 0.008 \\
Operative time & $1.212(0.397-3.701)$ & 0.762 \\
No. of instrumented level & $3.587(2.206-5.833)$ & $<0.001$ \\
Decompression & $2.106(0.268-16.534)$ & 0.702 \\
History of previous surgery & $7.455(2.447-22.711)$ & $<0.001$ \\
Hemovac & $2.323(0.297-18.196)$ & 0.702 \\
\hline OR, odds ratio; CI, confidence interval; ASA, American Society \\
of Anesthesiologists. & &
\end{tabular}

phylococcal infection was found in 7 patients. When 2 groups are compared using chi-square or Fisher exact test, total infection rate and total staphylococcal infection rate showed statistically significant difference $(p=0.028$ and $p=0.041$, respectively). The infection rates and statistical analysis are shown in Table 3.

\section{Outcome}

After surgery, perioperative complications regarding neuritis, cerebrospinal fluid leakage, pseudoarthrosis rate, implant failure, return to the operating room did not show significant difference between the 2 groups. Meanwhile, common adverse effect of vancomycin powder such as hypotension and renal toxicity was not observed in Vanco group. 
Table 5. Multivariate statistical analysis of potential risk factors

\begin{tabular}{lcc}
\hline \hline Variable & OR (95\% CI) & p-value \\
\hline Obesity & $5.767(0.875-37.999)$ & 0.069 \\
Diabetes mellitus & $18.789(2.254-156.632)$ & 0.007 \\
History of cardiovascular disease & $10.700(1.416-80.850)$ & 0.022 \\
Hospital stay & $1.449(1.122-1.870)$ & 0.004 \\
Blood loss & $4.506(0.676-30.055)$ & 0.120 \\
No. of instrumented level & $2.521(1.100-5.775)$ & 0.029 \\
History of previous surgery & $7.156(1.040-49.257)$ & 0.046 \\
\hline
\end{tabular}

OR, odds ratio; CI, confidence interval.

\section{Risk Factors}

In the univariate analysis of potential risk factors for acute SSIs among Vanco group, statistically significant risk factors were obesity (odds ratio [OR], 3.714; 95\% confidence interval [CI], 1.232-11.196), diabetes mellitus (OR, 4.958; 95\% CI, 1.701-14.455), history of cardiovascular disease (OR, 3.429; 95\% CI, 1.197-9.822), length of hospital stay (OR, 1.296; 95\% CI, 1.176-1.429), loss of blood during surgery over 1,000 mL (OR, 4.500; 95\% CI, 1.490-13.591), number of instrumented levels (OR, 3.587; 95\% CI, 2.206-5.833) and history of previous surgery (OR, 7.455; 95\% CI, 2.447-22.711).

In the multivariate analysis using binary logistic regression model, diabetes mellitus (OR, 18.787; 95\% CI, 2.254-156.632), history of cardiovascular disease (OR, 10.700; 95\% CI, 1.416$80.850)$, length of hospital stay (OR, 1.449 ; 95\% CI, 1.1221.870 ), number of instrumented levels (OR, 2.521; 95\% CI, $1.100-5.775)$ and history of previous surgery (OR, 7.156; 95\% CI, 1.040-49.257) were statistically significant risk factors increasing the risk of acute SSIs. Detailed univariate and multivariate statistical analysis are presented in Tables 4 and 5, respectively.

\section{DISCUSSION}

\section{Incidence of SSIs}

Our study was designed to clarify incidence, risk factors and outcome of SSIs between intrawound vancomycin applied group and nonvancomycin group. Our incidence of overall SSIs was $7.7 \%$ (44 of 571 ): Vanco group 5.5\% (15 of 275) and non-Vanco group $9.8 \%$ (29 of 296). Though addition of superficial infection to analysis might have influenced infection rate of our study higher than others, this rate is in line with other reports which reported incidence of SSIs between $2.8 \%$ and $10 \%$ after instrumented thoracolumbar spine fusion without use of topical vancomycin powder ${ }^{1,6,12,19,24)}$.

When limiting the result to the deep infection rate, study done by Scoliosis Research Society Morbidity and Mortality Committee reported deep infection rate of 1.3\% among 108,419 surgical cases though adjunctive measures for infection prophylaxis were not considered ${ }^{23)}$. Molinari et al. ${ }^{14)}$ reported deep wound infection rate of $1.2 \%$ for instrumented spinal surgery and $0.82 \%$ for uninstrumented surgeries. One of most meaningful studies done by Sweet et al. ${ }^{25}$ ) have shown deep infection rate of $2.6 \%$ among non-Vanco group compared to $0.2 \%$ in Vanco group though result is limited to posterior instrumented thoracic and lumbar spinal arthrodesis. As in other studies, our study demonstrated statistically significant decrement in postoperative deep infection rates with application of intrawound vancomycin powder.

\section{Mechanism of Intrawound Vancomycin Powder}

One of the important causes for postoperative spinal infection is intraoperative contamination of wounds ${ }^{11)}$. Hence, antibiotics and surgical wound irrigation have been a routine perioperative measures throughout various hospitals. In addition, to reduce SSI, various techniques including topical wound antibiotic instillation, closed wound suction drainage, shaving, silver dressings and wound irrigation with $0.35 \%$ povidoneiodine solution have been tested. None have proved superior to others except the use of betadine solution which showed some moderate evidence in preventing $\mathrm{SSI}^{22}$.

Lately, the efficacy of prophylactic intraoperative instillation of vancomycin is becoming a matter of interest for many clinicians undergoing spinal surgery due to emerging spinal infection. Vancomycin has been selected as prophylactic topical antibiotic of choice due to its cost-effectiveness, easy-to-use powdered form, and its effective broad coverage against organisms such as MRSA.

Sweet et al. $^{25)}$ have demonstrated that intrawound vancomycin powder is not readily absorbed into the systemic circulation, but rather stays in the wound and acts locally to prevent infection. Also, Gans et al. ${ }^{5)}$ reported systemic level of vancomycin lower than $2.0 \mu \mathrm{g} / \mathrm{mL}$ in 49 out of 50 patients which is lower than level required to cause systemic toxicitiy. This may be largely due to large molecular size of vancomycin, thus interrupting vancomycin from absorbed systematically ${ }^{7}$. Zebala et al. ${ }^{28)}$ conducted in vivo rabbit study by comparing vancomycin and control group which injected $S$. aureus into the wound after surgery and concluded that intrawound vancomycin concentration was high enough to defeat known staphylococcal infection.

Our study supports conclusions of above mentioned studies because reduction of SSI was effectively achieved and no side effect due to vancomycin was observed.

\section{Risk Factors for SSIs}

Risk factors for SSI in spine surgery have long been a matter of interest and can be divided into patient factors and surgical factors $4,5,7,12,17,24)$. Patient risk factors include advanced patient age, obesity, diabetes, urinary incontinence, smoking, and mal- 
nutrition. Surgical risk factors are composed of revision surgery, increased surgical blood loss, prolonged surgical time, use of instrumentation, and multilevel posterior fusions.

In our study, risk factors associated with development of SSI were diabetes mellitus, hypertension, history of previous surgery, length of hospital stay, and level of instrumentation. In context of patient factors, diabetes, hypertension, history of previous surgery, and length of hospital stay were statistically significant factors. Surgical factor was number of level instrumented.

\section{Limitations}

Our study has a limitation in that it is retrospectively designed. Lack of measurement in blood/wound vancomycin level could be weakness of our study. Also, deep MRSA and deep staphylococcal infection rates failed to show statistically significant reduction between the Vanco and non-Vanco groups. This may well be explained by small sample size. Thus, further investigation on larger group study should be conducted to confirm the actual difference between the 2 groups.

\section{CONCLUSION}

In this series of 571 patients, intrawound vancomycin powder usage resulted in significant decrease in SSI rates in our posterior lumbar surgical procedures. Presence of diabetes mellitus, cardiovascular disease, and longer hospital stay were statistically significant risk factors associated with SSI. Patients at high risk of infection are highly recommended as a candidate for this technique.

\section{CONFLICT OF INTEREST}

No potential conflict of interest relevant to this article was reported.

\section{REFERENCES}

1. Borkhuu B, Borowski A, Shah SA, Littleton AG, Dabney KW, Miller F: Antibiotic-loaded allograft decreases the rate of acute deep wound infection after spinal fusion in cerebral palsy. Spine (Phila Pa 1976) 33:2300-2304, 2008

2. Collins I, Wilson-MacDonald J, Chami G, Burgoyne W, Vineyakam P, Berendt T, et al: The diagnosis and management of infection following instrumented spinal fusion. Eur Spine J 17:445450, 2008

3. Engemann JJ, Carmeli Y, Cosgrove SE, Fowler VG, Bronstein $\mathrm{MZ}$, Trivette SL, et al: Adverse clinical and economic outcomes attributable to methicillin resistance among patients with Staphylococcus aureus surgical site infection. Clin Infect Dis 36: 592-598, 2003

4. Fang A, Hu SS, Endres N, Bradford DS: Risk factors for infection after spinal surgery. Spine (Phila Pa 1976) 30:1460-1465, 2005
5. Gans I, Dormans JP, Spiegel DA, Flynn JM, Sankar WN, Campbell RM, et al: Adjunctive vancomycin powder in pediatric spine surgery is safe. Spine (Phila Pa 1976) 38:1703-1707, 2013

6. Hodges SD, Humphreys SC, Eck JC, Covington LA, Kurzynske NG: Low postoperative infection rates with instrumented lumbar fusion. South Med J 91:1132-1136, 1998

7. Humphrey JS, Mehta S, Seaber AV, Vail TP: Pharmacokinetics of a degradable drug delivery system in bone. Clin Orthop Relat Res 349:218-224, 1998

8. Khan NR, Thompson CJ, DeCuypere M, Angotti JM, Kalobwe E, Muhlbauer MS, et al: A meta-analysis of spinal surgical site infection and vancomycin powder. J Neurosurg Spine 21:974983, 2014

9. Kirkland KB, Briggs JP, Trivette SL, Wilkinson WE, Sexton DJ: The impact of surgical-site infections in the 1990s: attributable mortality, excess length of hospitalization, and extra costs. Infect Control Hosp Epidemiol 20:725-730, 1999

10. Lee DG, Park KB, Kang DH, Hwang SH, Jung JM, Han JW: A clinical analysis of surgical treatment for spontaneous spinal infection. J Korean Neurosurg Soc 42:317-325, 2007

11. Lonstein J, Winter R, Moe J, Gaines D: Wound infection with Harrington instrumentation and spine fusion for scoliosis. Clin Orthop Relat Res 96:222-233, 1973

12. Massie JB, Heller JG, Abitbol JJ, McPherson D, Garfin SR: Postoperative posterior spinal wound infections. Clin Orthop Relat Res 284:99-108, 1992

13. McLeod LM, Keren R, Gerber J, French B, Song L, Sampson $\mathrm{NR}$, et al: Perioperative antibiotic use for spinal surgery procedures in US children's hospitals. Spine (Phila Pa 1976) 38:609616, 2013

14. Molinari RW, Khera OA, Molinari WJ 3rd: Prophylactic intraoperative powdered vancomycin and postoperative deep spinal wound infection: 1,512 consecutive surgical cases over a 6-year period. Eur Spine J 21 Suppl 4:S476-482, 2012

15. Muilwijk J, Walenkamp GH, Voss A, Wille JC, van den Hof S: Random effect modelling of patient-related risk factors in orthopaedic procedures: results from the Dutch nosocomial infection surveillance network 'PREZIES'. J Hosp Infect 62:319-326, 2006

16. O'Neill KR, Smith JG, Abtahi AM, Archer KR, Spengler DM, McGirt MJ, et al: Reduced surgical site infections in patients undergoing posterior spinal stabilization of traumatic injuries using vancomycin powder. Spine J 11:641-646, 2011

17. Olsen MA, Mayfield J, Lauryssen C, Polish LB, Jones M, Vest $\mathrm{J}$, et al: Risk factors for surgical site infection in spinal surgery. J Neurosurg 98(2 Suppl):149-155, 2003

18. Olsen MA, Nepple JJ, Riew KD, Lenke LG, Bridwell KH, Mayfield J, et al: Risk factors for surgical site infection following orthopaedic spinal operations. J Bone Joint Surg Am 90:62-69, 2008

19. Rechtine GR, Bono PL, Cahill D, Bolesta MJ, Chrin AM: Postoperative wound infection after instrumentation of thoracic and lumbar fractures. J Orthop Trauma 15:566-569, 2001

20. Sasso RC, Garrido BJ: Postoperative spinal wound infections. J Am Acad Orthop Surg 16:330-337, 2008

21. Schimmel JJ, Horsting PP, de Kleuver M, Wonders G, van Limbeek J: Risk factors for deep surgical site infections after spinal fusion. Eur Spine J 19:1711-1719, 2010

22. Schuster JM, Rechtine G, Norvell DC, Dettori JR: The influence 
of perioperative risk factors and therapeutic interventions on infection rates after spine surgery: a systematic review. Spine (Phila Pa 1976) 35(9 Suppl):S125-137, 2010

23. Smith JS, Shaffrey CI, Sansur CA, Berven SH, Fu KM, Broadstone PA, et al: Rates of infection after spine surgery based on 108,419 procedures: a report from the Scoliosis Research Society Morbidity and Mortality Committee. Spine (Phila Pa 1976) 36: 556-563, 2011

24. Sponseller PD, LaPorte DM, Hungerford MW, Eck K, Bridwell $\mathrm{KH}$, Lenke LG: Deep wound infections after neuromuscular scoliosis surgery: a multicenter study of risk factors and treatment outcomes. Spine (Phila Pa 1976) 25:2461-2466, 2000

25. Sweet FA, Roh M, Sliva C: Intrawound application of vancomycin for prophylaxis in instrumented thoracolumbar fusions: efficacy, drug levels, and patient outcomes. Spine (Phila Pa 1976)
36:2084-2088, 2011

26. Tubaki VR, Rajasekaran S, Shetty AP: Effects of using intravenous antibiotic only versus local intrawound vancomycin antibiotic powder application in addition to intravenous antibiotics on postoperative infection in spine surgery in 907 patients. Spine (Phila Pa 1976) 38:2149-2155, 2013

27. Williams DN, Gustilo RB, Beverly R, Kind AC: Bone and serum concentrations of five cephalosporin drugs. Relevance to prophylaxis and treatment in orthopedic surgery. Clin Orthop Relat Res 179:253-265, 1983

28. Zebala LP, Chuntarapas T, Kelly MP, Talcott M, Greco S, Riew $\mathrm{KD}$ : Intrawound vancomycin powder eradicates surgical wound contamination: an in vivo rabbit study. J Bone Joint Surg Am 96:46-51, 2014 